ÉGYPTE monde arabe

\section{Égypte/Monde arabe}

18-19| 1994

L'éducation en Égypte

\title{
L'éducation féminine en Égypte
}

Éléments pour un bilan

\section{Nader Fergany}

\section{(2) OpenEdition}

\section{Journals}

Édition électronique

URL : https://journals.openedition.org/ema/85

DOI : 10.4000/ema. 85

ISSN : 2090-7273

Éditeur

CEDEJ - Centre d'études et de documentation économiques juridiques et sociales

Édition imprimée

Date de publication : 30 septembre 1994

Pagination : 101-113

ISSN : 1110-5097

Référence électronique

Nader Fergany, «L'éducation féminine en Égypte », Égypte/Monde arabe [En ligne], 18-19 | 1994, mis en ligne le 08 juillet 2008, consulté le 07 juillet 2022. URL : http://journals.openedition.org/ema/85 ; DOI : https://doi.org/10.4000/ema.85

Ce document a été généré automatiquement le 7 juillet 2022

Tous droits réservés 


\title{
L'éducation féminine en Égypte
}

\author{
Éléments pour un bilan
}

Nader Fergany

1 Cette étude ${ }^{1}$ tente d'évaluer les résultats enregistrés dans le domaine de l'éducation des Egyptiennes au début des années 90 , soit 120 ans après qu'a été entamée la scolarisation institutionnalisée des jeunes filles. L'Égypte affronte aujourd'hui l'important défi cognitif et technique que lui posent les pays industrialisés. Alors que les secteurs avancés de la civilisation occidentale traversent aujourd'hui une étape décisive dans l'histoire des savoirs et des cultures, l'Égypte se débat toujours dans une phase industrielle primaire. Elle n'est pas encore parvenue à éradiquer l'analphabétisme ni à en enrayer l'augmentation en donnant à ses citoyens une scolarisation de base, sans parier de la qualité de l'enseignement qui ne sera pas abordée ici.

2 L'accès à l'éducation est loin d'être harmonieusement réparti en Égypte. Il existe dans ce domaine des régions mal loties, des catégories sociales vulnérables; les femmes, en particulier, pâtissent d'un processus de marginalisation aisément observable dans les formations sociales les moins avancées.

3 La lutte contre l'analphabétisme est bien sûr prioritaire. Avant d'en évaluer l'étendue à la lumière des données les plus récentes et les plus exhaustives, soit le recensement de 1986, il nous faut préciser que ce dernier considère comme alphabétisées les personnes ayant suivi quatre années complètes d'enseignement primaire, ce qui nous semble pêcher par excès de générosité. Cela revient en effet à supposer acquis, en quatre ans, les éléments de base de l'éducation, hypothèse pour le moins contestable comme le montre l'une des rares enquêtes - sinon la seule - effectuées sur la qualité de l'enseignement. Non seulement le cycle primaire, selon cette enquête, ne suffit pas à assurer un niveau d'alphabétisation fonctionnel, mais on observe encore, à cette étape, un niveau moindre chez les filles que chez les garçons. En d'autres termes, le recensement sous-estime le degré d'analphabétisme, notamment en ce qui concerne les femmes. 
Tableau 1 : Pourcentage d'analphabétisme par sexe et région (urbaine/rurale) Recensement de 1986

\begin{tabular}{|l|l|l|l|}
\hline Lieu de résidence & \multicolumn{4}{|l|}{ Sexe } \\
\hline & Hommes & Femmes & Total \\
\hline Urbain & 27 & 45 & 35 \\
\hline Rural & 47 & 76 & 61 \\
\hline Total & 38 & 62 & 50 \\
\hline
\end{tabular}

4 Les résultats du recensement indiquent qu'au milieu des années 80 - toujours d'après une définition très large de l'analphabétisme -, la moitié de la population au moins est constituée d'analphabètes et le phénomène frappe les femmes davantage que les hommes, surtout en milieu rural : il en atteint les quatre cinquièmes. Selon une étude plus fine, l'analphabétisme des femmes de milieu rural est plus élevé dans la vallée du Nil que dans le Delta.

5 L'observation des chiffres dans les 432 districts urbains (qism) et ruraux (markaz) que compte l'Égypte montre que toutes les localités pâtissent de l'analphabétisme féminin. Dans les zones rurales, où il est le plus élevé, il est au minimum de $50 \%$, mais il atteint $100 \%$ dans certaines zones urbaines, comme l'indique le graphique suivant :

L'analphabétisme féminin dans les zones urbaines et rurales

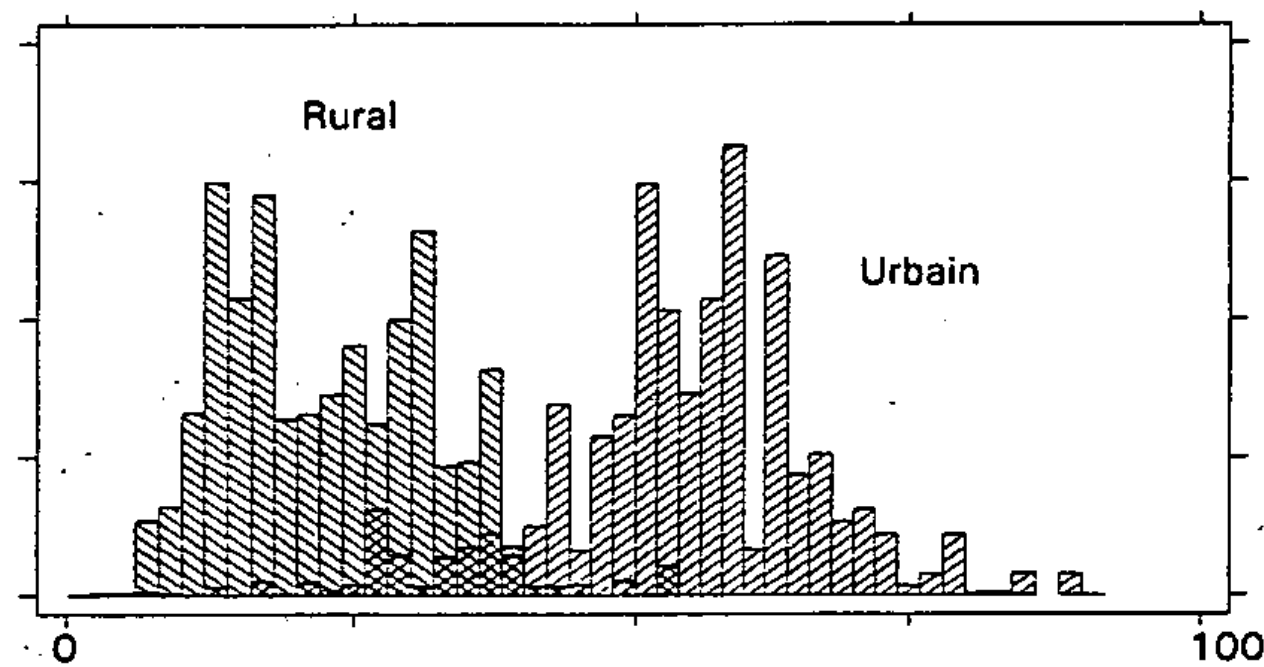

6 Si cent vingt années de scolarisation des femmes aboutissent à un bilan aussi désastreux, ne serait-ce qu'en termes d'alphabétisation, on peut se demander en combien d'années sera éradiqué l'analphabétisme féminin... Au risque, de choquer, posons une hypothèse: plus de 70 ans pour le Caire, et plus de... 500 ans pour les régions rurales de la Vallée! 


\section{Le taux de fréquentation scolaire dans l'enseignement primaire}

7 L'Égypte est sans doute un des rares pays au monde qui ait décidé, il y a quelques années, de réduire - de 6 à 5 ans - le cycle primaire, mesure qui serait compréhensible si cet enseignement dépassait en qualité tous les standards connus. Or, l'enseignement primaire étant le premier rempart contre l'analphabétisme, ses carences participent activement à son aggravation.

8 Les pourcentages de scolarisation dans le cycle primaire entre 6 et 11 ans, présentés ici par sexe et par région, sont issus d'une enquête nationale réalisée en 1988 (Fergani, 1993). Celle-ci analyse les taux de fréquentation scolaire masculine et féminine dans huit régions d'Égypte en privilégiant le découpage urbain/rural et, à un deuxième niveau, le découpage villages/fermes et hameaux;

Tableau 2 : Taux de fréquentation scolaire des élèves de 6 à 11 ans (par sexe et par région) octobre 1988

\begin{tabular}{|l|l|l|l|}
\hline Lieu de résidence & \multicolumn{3}{|l|}{ Taux de fréquentation } \\
\hline & Garçons & Filles & Total \\
\hline Le Grand-Caire & 98 & 95 & 97 \\
\hline Alexandrie. Suez, Port-Saïd, Ismaîliya & 96 & 91 & 94 \\
\hline Delta urbain & 97 & 97 & 97 \\
\hline Haute-Égypte urbaine & 95 & 94 & 95 \\
\hline Delta (villages) & 94 & 78 & 86 \\
\hline Delta (fermes/hameaux) & 92 & 78 & 85 \\
\hline Haute-Égypte (villages) & 89 & 65 & 78 \\
\hline Haute-Égypte (fermes/hameaux) & 89 & 57 & 73 \\
\hline Égypte & 94 & 80 & 87 \\
\hline
\end{tabular}

9 L'on peut en tirer certaines conclusions.

10 Tout d'abord, c'est en milieu rural que la scolarisation dans le cycle primaire est la plus faible, notamment dans la Vallée et au sein de celle-ci, dans les fermes et hameaux. Alors que ce taux, dans les zones urbaines de la Vallée et du Delta, atteint près de $95 \%$, il ne dépasse pas $85 \%$ dans les villages et hameaux du Delta, $78 \%$ dans les villages de la Vallée et $73 \%$ dans les hameaux de la Vallée. Ce mode de répartition soulève la question du faible peuplement des fermes et hameaux, surtout dans la Vallée, état de fait qui rend coûteuse et inadéquate la mise en place d'équipements scolaires standard. 
Fig. 2 : Évaluation du taux de fréquentation de la classe d'âge (6-11 ans) en fonction des régions et du sexe. octobre 1988

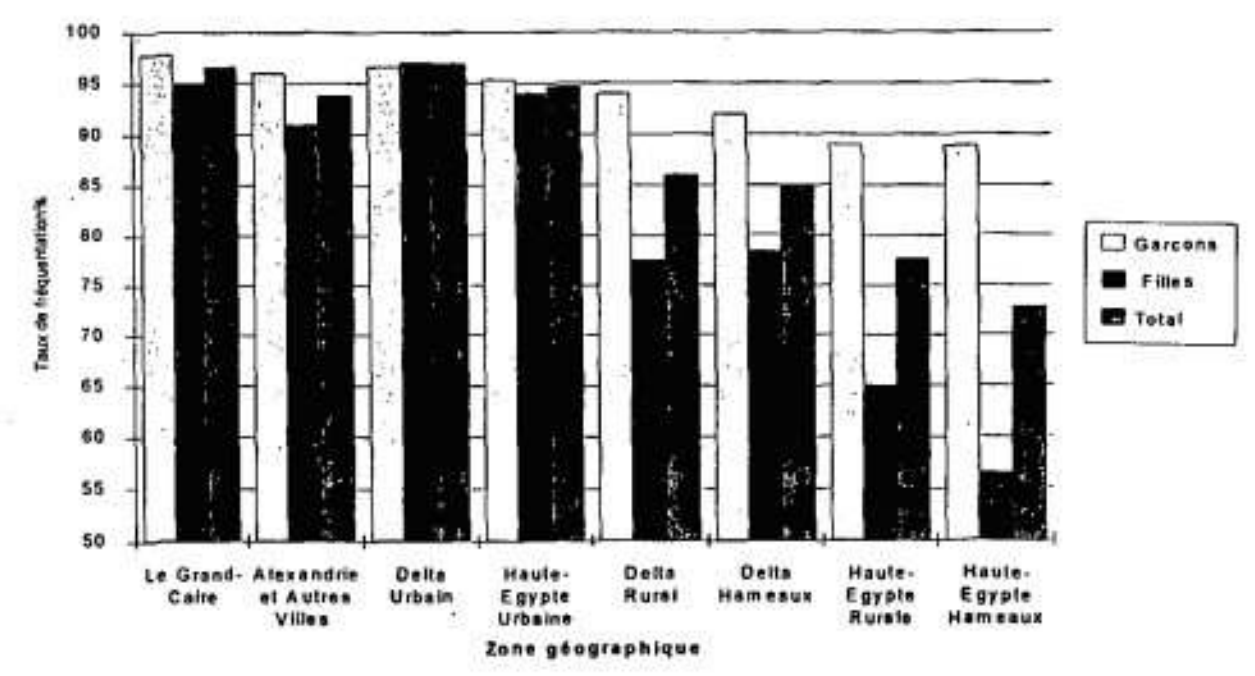

11 Lorsque l'écart se creuse entre régions, cela est dû essentiellement au taux de scolarisation féminine : alors qu'il oscille autour de $95 \%$ dans les zones urbaines du Delta et de la Vallée, sans différence significative avec le taux masculin - ce qui donne des taux équivalents à ceux du Grand Caire - le taux de scolarisation des filles dans les villages et les hameaux du Delta ne dépasse pas $78 \%$ et tombe à $65 \%$ dans les villages de la Vallée. Le taux de scolarisation des filles se limite à $57 \%$ dans les hameaux de la Vallée.

12 L'écart des taux de scolarisation respectifs des filles et des garçons est sensible entre les zones rurales du Delta et celles de la Vallée. Dans les zones rurales de la Vallée, on peut également repérer un écart entre villages et fermes. Le taux de scolarisation des garçons dans les zones rurales de la Vallée atteint près de $90 \%$; en d'autres termes, dans ces mêmes régions, celui des filles, nettement inférieur, dépend de facteurs importants tels que la proximité d'équipements scolaires dans les zones peu peuplées. Alors que les garçons peuvent parcourir une distance relativement longue jusqu'à l'école, les milieux conservateurs seront réticents à le permettre aux filles.

13 Ces premières indications montrent combien il importe d'étudier de près les modes d'accès au primaire et les facteurs socio-économiques qui pèsent sur le taux de scolarisation, en particulier sur celui des filles. Ceci nous semble constituer le fondement de toute politique visant à la diffusion de l'enseignement en Égypte. Encore une fois, si les taux de scolarisation en vigueur durant les cent vingt dernières années demeurent tels, dans combien d'années la scolarisation primaire des hameaux de la Vallée sera-t-elle assurée ? Plus de 90 ans !

14 L'enquête mentionnée ci-dessus a par ailleurs tenté d'évaluer le poids des divers facteurs socio-économiques qui conditionnent l'accès à l'enseignement. En tête des facteurs qui entravent la scolarisation au niveau du primaire vient le travail des enfants, notamment quand il se situe hors du cercle familial. Les (acteurs d'ordre social sont plus déterminants s'agissant de la scolarisation des filles, notamment dans certaines régions (zones rurales de la Vallée ou de l'ouest du Delta).

15 Autre variable déterminante du taux de scolarisation sur le plan local; le niveau d'instruction au sein de la famille, notamment celui du chef de famille et des femmes 
adultes. En comparaison avec cette variable, le poids du niveau économique de la famille ou de la région semble moins déterminant. Autant d'indices qui montrent que le taux de scolarisation s'articule aux caractéristiques d'un sous-développement qu'il semble difficile de dépasser à court terme.

Fig. 3 : Alphabétisation des femmes

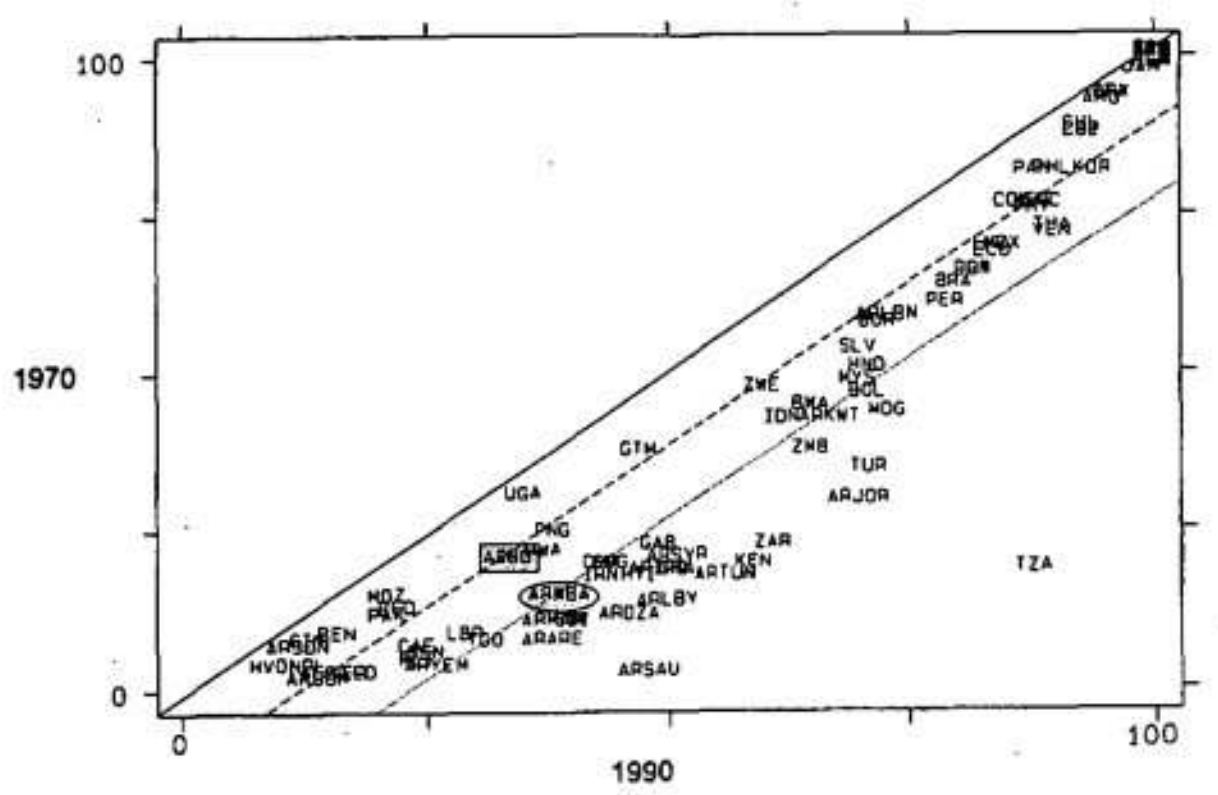

Fig. 4 : Alphabétisation comparée des femmes et des hommes

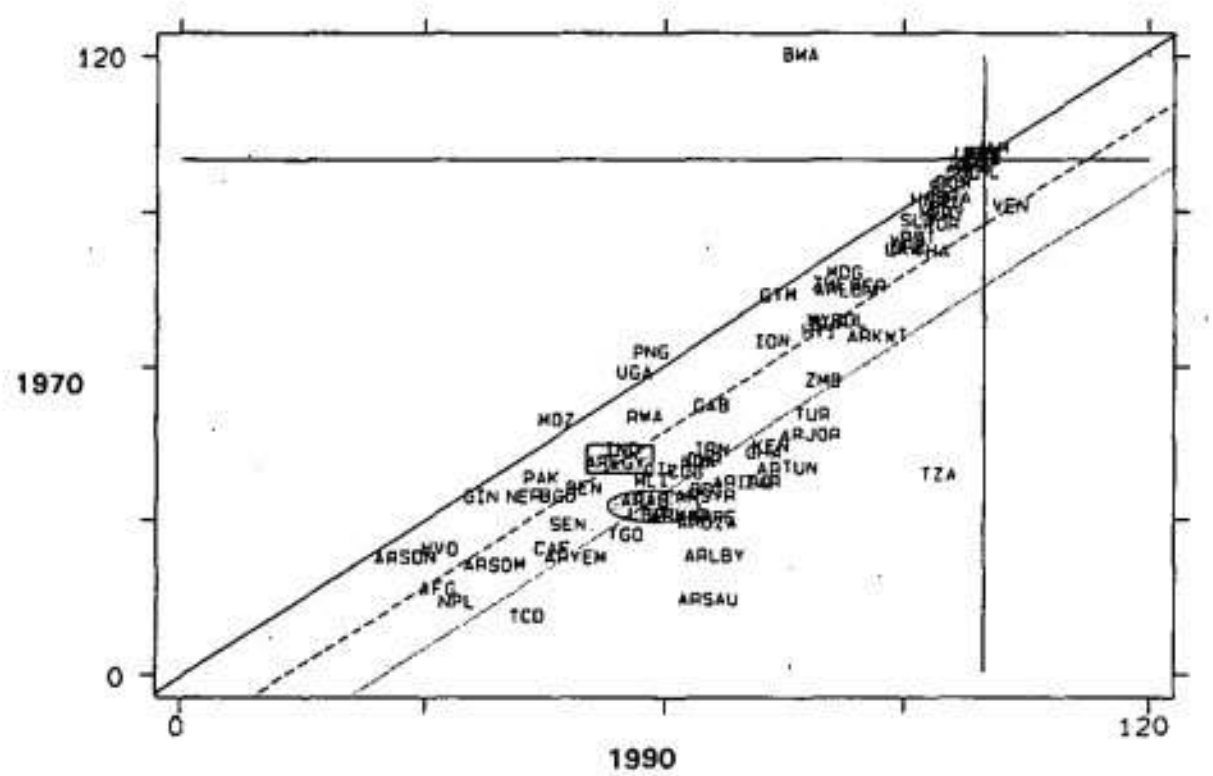

16 Rappelons enfin que nous ne disposons pas de données fiables et récentes sur la qualité de l'enseignement primaire ou, en d'autres termes, d'une évaluation scientifique exacte 
du niveau d'acquisition des connaissances de base (lecture, écriture, mathématiques) de l'élève en Égypte. La seule étude dans ce domaine (Hartley \& Swanson, 1988) a été menée par la Banque mondiale et le Centre de recherches pédagogiques au début des années 80 ; elle montre l'insuffisance du niveau des connaissances de base acquises par ceux qui achèvent le cycle primaire. L'analphabétisme est donc fatal pour ceux qui quittent l'école, même après six ans de scolarisation. Selon cette même étude, le niveau d'acquisition de ces derniers est déjà nettement inférieur à celui de leurs condisciples. En outre, le niveau des filles est généralement plus faible que celui des garçons, notamment pour ce qui est des mathématiques. La conjugaison de ces résultats montre bien que la marginalisation sociale est un des principaux déterminants du niveau d'acquisition des connaissances de base dans le cycle primaire.

\section{L'éducation des femmes à l'échelle mondiale : un regard comparatif}

17 Les pays sous-développés tendent souvent à comparer leur état présent à leur état antérieur pour s'accorder des satisfecits à peu de frais. Il n'empêche que la mise en situation la plus pertinente, dans une perspective de développement, est celle qui prend en compte certains critères - ici, ceux relatifs à l'éradication de l'analphabétisme ou la diffusion de l'enseignement primaire à l'échelle nationale. Une autre comparaison pourrait être tentée entre l'Égypte et d'autres pays. Une représentation graphique de la progression temporelle relative sur les trente dernières années (voir en annexe) permet de le faire. Elle se base sur des données indicatives fournies par les instances internationales (Banque mondiale, Unicef) et qui suffisent largement aux objectifs de cette démonstration. Les graphiques 3 à 7 représentent la position relative de l'Égypte dans les domaines suivants :

- alphabétisation des femmes (graph. 3) ;

- alphabétisation comparée des femmes et des hommes (graph. 4) :

- inscription des filles dans l'enseignement primaire (graph. 5);

- inscription comparée des filles et des garçons dans l'enseignement primaire (graph. 6) ;

- inscription des filles dans le cycle secondaire (graph. 7).

18 (Sur les graphiques reproduits dans ces pages, l'Égypte est représentée par un rectangle et l'ensemble des pays arabes par une forme ovale). 
Fig. 5 : Inscription des filles dans l'enseignement primaire

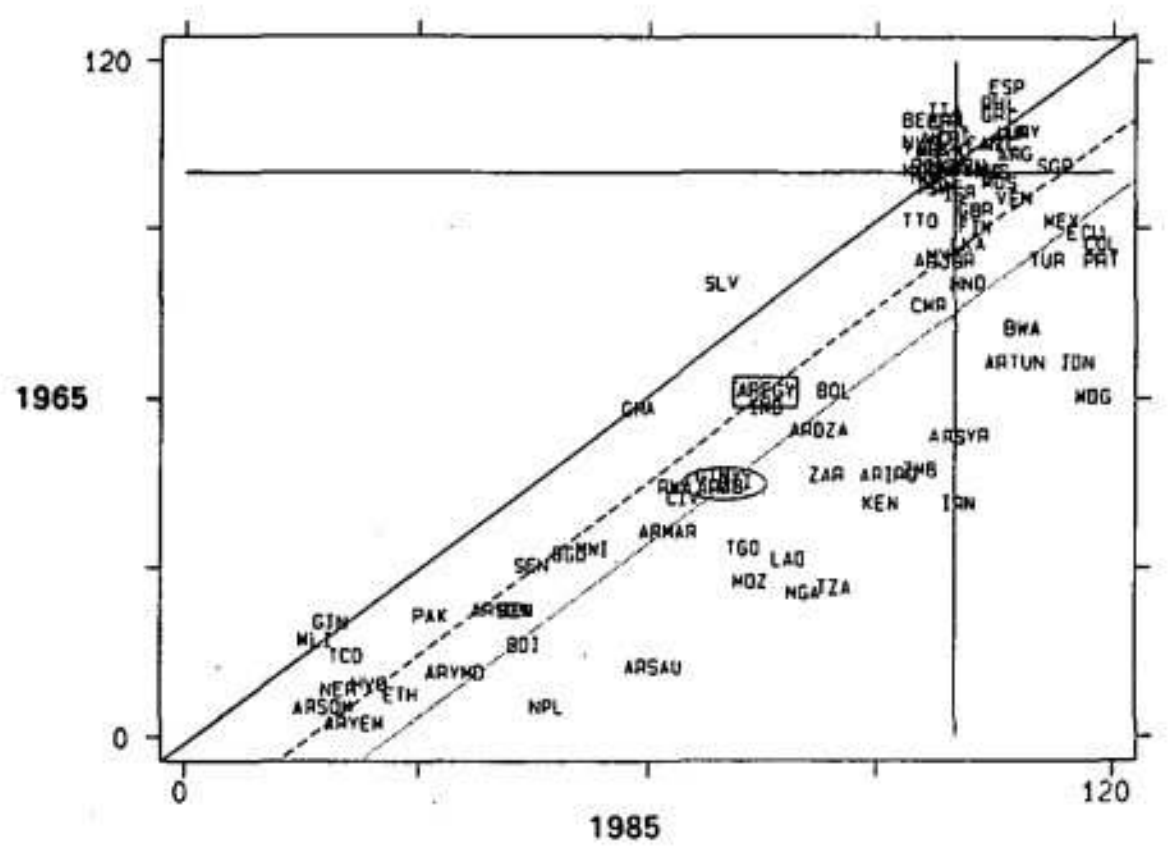

Fig. 6 : Inscription comparée des filles et des garçons dans l'enseignement primaire

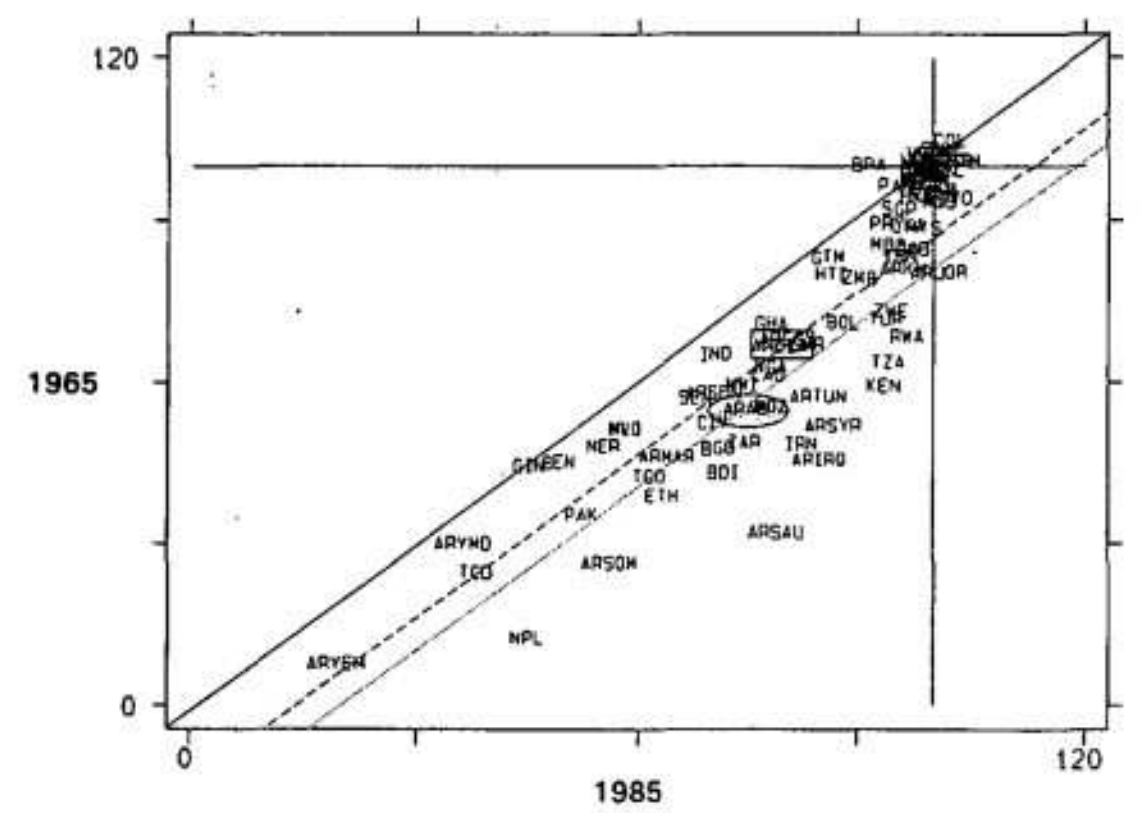

19 Les graphiques 3 et 6 permettent de dégager deux conclusions principales : la majeure partie des pays du tiers-monde a réalisé des progrès en matière d'alphabétisation des femmes et de réduction des écarts entre hommes et femmes dans ce domaine, cela à 
des taux supérieurs à ceux enregistrés en Égypte. En outre, un grand nombre de pays qui se classaient après l'Égypte l'ont rattrapée et dépassée durant la période considérée. Par ailleurs, l'ensemble des pays arabes a également progressé dans ce domaine. Là aussi les taux de scolarisation sont plus élevés que ceux de l'Égypte, y compris pour un grand nombre de pays qui lui ont succédé et qui, aujourd'hui, la devancent en matière d'éradication de l'analphabétisme.

Ces performances affligeantes ne se retrouvent pas dans les stades supérieurs de l'enseignement. En ce qui concerne l'accroissement de la scolarisation des filles dans le secondaire, l'Égypte devance l'ensemble des pays arabes et la majorité des pays du tiers-monde (graph. 7 page suivante), phénomène qui traduit bien le déséquilibre existant dans la structure même du système éducatif égyptien : l'on met l'accent sur les cycles supérieurs pour satisfaire aux demandes des milieux les plus favorisés, cela aux dépens de l'enseignement de base, limite que ne franchissent pas les enfants des classes pauvres... quand bien même ils y parviennent.

21 Le résultat de ce retard proportionnel fait que l'Égypte se retrouve en position de retard absolu par rapport à l'ensemble des pays arabes et, dans un taux plus élevé, par rapport au reste du tiers-monde pour ce qui est de l'alphabétisation féminine.

Autrement dit, comparée à l'ensemble des pays arabes et au reste du monde, l'Égypte enregistre un recul dans le domaine de l'éradication de l'analphabétisme féminin et de la scolarisation des filles dans le cycle primaire.

N'est-il pas temps de sonner l'alarme?

\section{Conclusion}

24 Aujourd'hui, il semble admis que le seul développement durable est celui des ressources humaines. Tous les pays qui ont réalisé un développement rapide, comme ceux de l'Est asiatique, ont commencé par un investissement à large échelle et de haut niveau dans le capital humain ; santé, formation (et pas seulement éducation) et liberté. L'Égypte a enregistré d'importantes réalisations dans le secteur de la santé durant les trente dernières années. S'il reste encore beaucoup à faire dans ce domaine, les progrès réalisés pourront, semble-t-il, se poursuivre sans heurts notoires. La crise aiguë que traversent les pays sous-développés se manifeste surtout dans le domaine du savoir, ce qui renvoie tant aux carences du système éducatif qu'à la conjoncture sociale qui les accompagne. L'enseignement élémentaire demeure la pierre angulaire de tout développement des ressources humaines. Dans cette perspective, il faut, au minimum, aspirer à promouvoir, à l'échelle nationale, un enseignement primaire puis élémentaire de qualité et la mise en place des dispositifs qui favorisent la créativité dans le processus éducatif. Ces objectifs ne pourront être réalisés tant que durera la marginalisation des catégories sociales les plus vulnérables, à commencer par les femmes, tant dans le processus éducatif qu'à l'échelle globale de la société. 
Fig. 7 : Inscription des filles dans le cycle secondaire

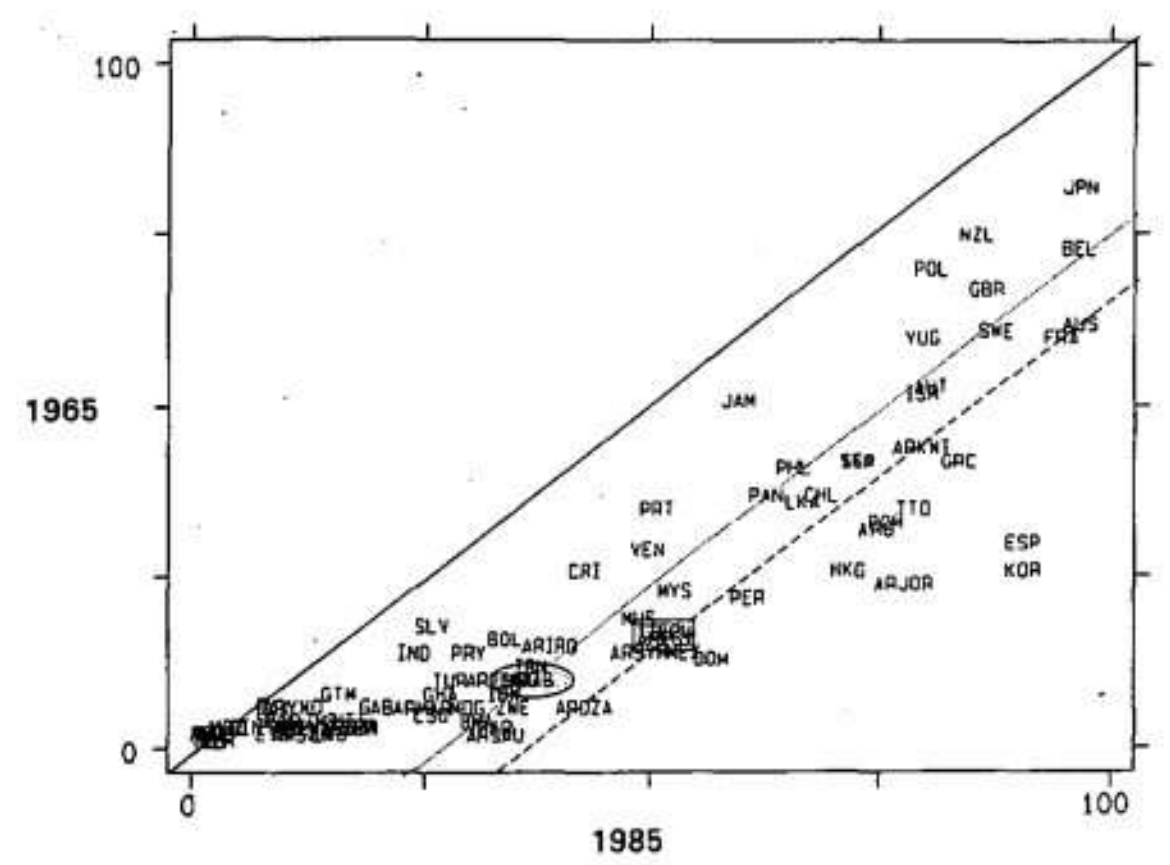

Tableau 3 : L'enseignement des femmes en Égypte comparé aux autres pays (pays arabes, tiersmonde, pays industrialisés)

\begin{tabular}{|c|c|c|c|c|c|}
\hline \multirow[t]{2}{*}{ Indicateurs } & \multirow[t]{2}{*}{ Égypte } & \multicolumn{4}{|c|}{ Moyenne } \\
\hline & & $\begin{array}{l}\text { Pays } \\
\text { arabes }\end{array}$ & $\begin{array}{l}\text { Autres pays du tiers- } \\
\text { monde }\end{array}$ & \multicolumn{2}{|c|}{\begin{tabular}{|l} 
Pays \\
industrialisés
\end{tabular}} \\
\hline $\begin{array}{l}\text { Taux d'alphabétisation des femmes en } \\
1970\end{array}$ & 20 & 14 & & 38 & 98 \\
\hline $\begin{array}{l}\text { Taux d'alphabétisation des femmes en } \\
1990\end{array}$ & 34 & 38 & & 56 & 99 \\
\hline $\begin{array}{l}\text { Taux de fréquentation des filles dans le } \\
\text { primaire } 1965\end{array}$ & 60 & 44 & & 63 & 106 \\
\hline $\begin{array}{l}\text { Taux de fréquentation des filles dans le } \\
\text { primaire } 1985\end{array}$ & 76 & 71 & & 93 & 101 \\
\hline
\end{tabular}




\section{BIBLIOGRAPHIE}

Fergany N., Anmât al-itfihâq bi-l-ta'lîm al-ibtidâ'i (Modes d'accès à l'enseignement primaire), Le Caire, al-Mishkah, 1993.

Hartley M \& Swanson E. V., Retention of Basic Skills among Dropouts from Egyptian Primary Schools, World Bank, Washington D. C., January 1988.

\section{ANNEXES}
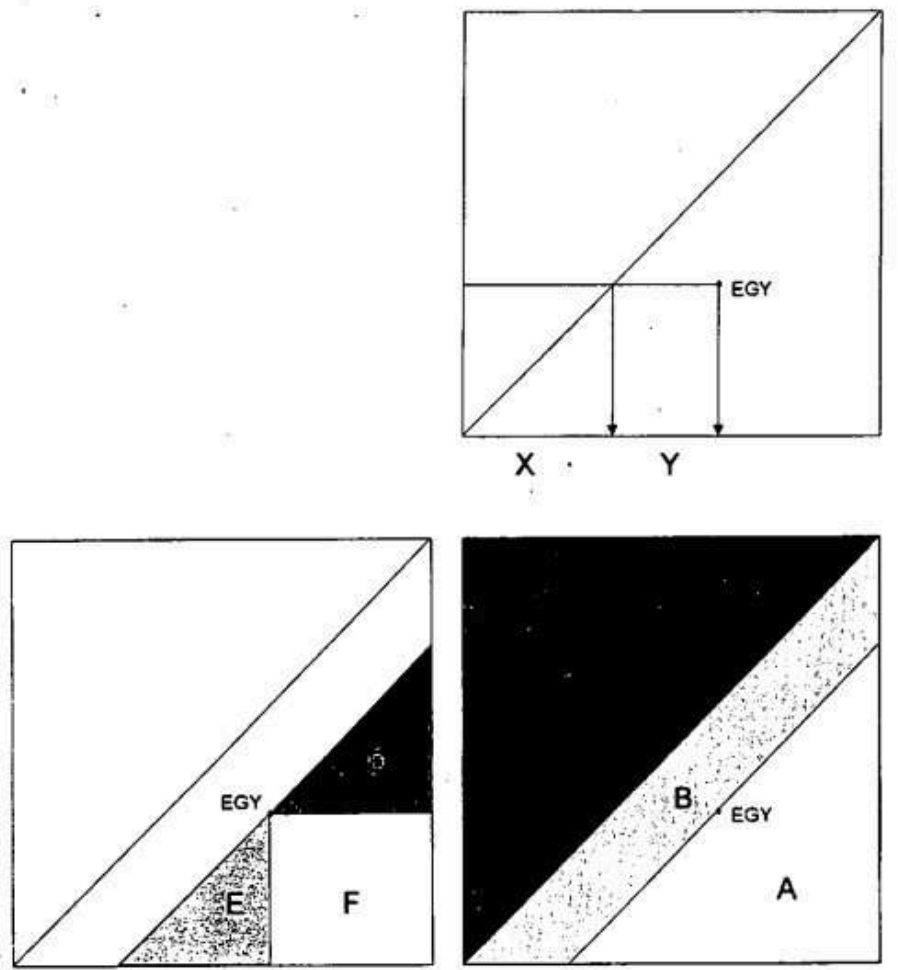

Annexe : progression temporelle relative

Les données de la période la plus récente sont présentées en abscisse et ceux de la période antérieure en ordonnée (voir graphique ci-contre).

Distance $\mathrm{X}$ : valeur de la variable en Égypte au début de la période considérée.

Distance $\mathrm{X}+\mathrm{Y}$ : valeur de la variable en Égypte à la fin de la période considérée.

Distance Y : amélioration absolue de la position de l'Égypte pendant la période considérée.

Zone $\mathrm{A}$ : pays dont la position s'est améliorée à un taux supérieur à celui de l'Égypte durant la période considérée.

Zone $\mathrm{B}$ : pays dont la position s'est améliorée à un taux inférieur à celui de l'Égypte durant la période considérée.

Zone C : pays dont la position s'est détériorée. 
Zone D ; pays dont la position était en avance par rapport à l'Égypte au début de la période considérée, et dont l'amélioration a été plus rapide.

Zone $\mathrm{E}$ : pays dont la position était en retrait par rapport à l'Égypte au début de la période considérée et qui restent en retrait à la fin de celle-ci, mais dont l'amélioration a suivi un rythme plus rapide.

Zone $\mathrm{F}$ : pays dont la position par rapport à l'Égypte était en retrait au début de la période considérée, et qui la devancent à la fin de celle-ci.

\section{NOTES}

1. Tiré de l'hebdomadaire al-Ahram al-Iqtisâdi, 10 janvier 1994, traduit de l'arabe par Iman Farag.

\section{INDEX}

Mots-clés : éducation, enseignement, enseignement féminin, genre, primaire, statistiques, femme

\section{AUTEUR}

\section{NADER FERGANY}

Center for Research and Training 